eISSN 2444-7986

DOI: https://doi.org/10.14201/orl.17348

Artículo de revisión

\title{
REVISIÓN SOBRE LA DESVIACIÓN OBLICUA
}

\section{Review of the skew deviation}

\author{
Eduardo Antonio MENA-DOMÍNGUEZ \\ Servicio de Otorrinolaringología. Hospital Santa Bárbara, Puertollano (Ciudad Real). España.
}

Correspondencia: emenad@sescam.jccm.es; edumen2@gmail.com

Fecha de recepción: 1 de diciembre de 2017

Fecha de aceptación: 5 de diciembre de 2017

Fecha de publicación: 8 de diciembre de 2017

Fecha de publicación del fascículo: 1 junio de 2018

Conflicto de intereses: Los autores declaran no tener conflictos de intereses

Imágenes: Los autores declaran haber obtenido las imágenes con el permiso de los pacientes

Politica de derechos y autoarchivo: se permite el autoarchivo de la versión post-print (SHERPA/RoMEO)

Licencia CC BY-NC-ND. Licencia Creative Commons Atribución-NoComercial-SinDerivar 4.0 Internacional

Universidad de Salamanca. Su comercialización está sujeta al permiso del editor

RESUMEN

Introducción: La desviación oblicua es una falta de alineación vertical de los ojos que no puede ser explicada como resultado de una parálisis de los músculos oculares. Revisaremos la fisiopatología de este síndrome, su expresividad clínica y la forma de diagnosticarlo. Métodos: Revisión narrativa. Resultados: La desviación oblicua es el resultado de una lesión en la vía neural vestíbulo-ocular. Al tratarse de una vía cruzada, en función de donde se localice la patología, la falta de alineamiento ocular puede afectar a uno $u$ otro globo ocular. Tanto en lesiones periféricas como centrales, puede acompañarse de inclinación lateral de la cabeza y de torsión ocular, lo que determina la triada conocida como "reacción de inclinación óculocefálica". Para el diagnóstico de la desviación oblicua, además de la observación utilizaremos el "cover test" y la varilla de Maddox. Discusión: Aunque la fisiopatología de estas manifestaciones clínicas resulta muy interesante, se trata de un proceso infrecuente, aún por comprender y con escaso valor localizador. Conclusión: La desviación oblicua siempre obliga a descartar, mediante pruebas de imagen, patología neurológica central. En el contexto de un vértigo agudo, puede ser el único indicador de afectación del sistema nervioso central.

PALABRAS CLAVE desviación oblicua; utrículo; vía otolítica; reacción inclinación ocular; vértigo; tronco cerebral

SUMMARY Introduction: Skew deviation is a lack of vertical alignment of the eyes that cannot be explained
as a result of paralysis of the eye muscles. We will review the pathophysiology of this syndrome,
its clinical expressivity and how to diagnose it. Methods: Narrative review. Results: The skew
deviation is the result of a lesion in the vestibular-ocular neural pathway. Being a cross-way,
depending on where the pathology is located, the lack of ocular alignment can affect one or the
other eyeball. In both peripheral and central lesions, it can be accompanied by lateral tilt of the
head and ocular torsion, which determines the triad known as "ocular-cephalic tilt reaction".
For the diagnosis of the skew deviation, in addition to the observation we will use the "cover
test" and the rod of Maddox. Discussion: Although the physiopathology of these clinical mani-
festations is very interesting, it is an infrequent process, still to be understood and with little
locator value. Conclusion: Skew deviation always requires discarding, through imaging tests, 
central neurological pathology. In the context of acute vertigo, it may be the only indicator of involvement of the Central Nervous System.

\section{INTRODUCCIÓN}

La skew deviation, que traducido al español significaría desviación oblicua, se manifiesta por una falta de alineamiento vertical de los ojos, que no puede ser explicado como resultado de una parálisis de los músculos oculares [1-5]. La característica más especial de este cuadro es el cambio que en ocasiones se observa en la posición de los ojos según la dirección de la mirada, de tal manera que el ojo puede estar en hipertropía (más elevado) cuando realiza la abducción y en hipotropía (más descendido) con la aducción, de donde deriva el nombre de desviación oblicua [6]. Cuando además se acompaña de inclinación lateral de la cabeza y torsión ocular, determina una tríada conocida como reacción de inclinación óculo-cefálica (ocular tilt reaction, OTR). No obstante, la comprensión de este proceso tan infrecuente, está todavía en evolución [1,3-5,7,8].

Este movimiento coordinado de cabeza y ojos es controlado por el sistema vestibular periférico, en respuesta al input sensorial bilateral que llega al utrículo, sáculo y conducto semicircular posterior (CSP) $[1,7,9,10]$. La vía responsable de la desviación oblicua es la otolítica-ocular, que desde el laberinto asciende ipsilateralmente al núcleo vestibular de la protuberancia, donde se decusa haciéndose contralateral para, a través del fascículo longitudinal medial, alcanzar los núcleos óculomotores, el núcleo intersticial de Cajal, el mesencéfalo y el tálamo $[11,12]$. El componente espinal, vestíbulo-cefálico, responsable de la inclinación de la cabeza es también de origen laberíntico y se asocia a una alteración subjetiva de la inclinación del plano vertical, de tal manera que el paciente mantiene el tortícolis para percibir lo que ahora es derecho para él [1].

En este capítulo, revisaremos la fisiopatología de este síndrome, su expresividad clínica y la forma de diagnosticarlo.

\section{ANTECEDENTES HISTÓRICOS}

La desviación oblicua fue identificada de manera independiente por Magendie y Hertwig en el siglo XIX, al provocar lesiones experimentales en el cerebelo y tronco del encéfalo (TE) del animal de experimentación [12]. Y aunque posteriormente se refiere en pacientes con diferentes lesiones neurológicas del cerebelo, TE, núcleos vestibulares e incluso en la otitis media crónica, no es hasta 1975 en que Westheimer y Blair describen los mecanismos de este estrabismo vertical y dan nombre a la reacción de inclinación (OTR). Al estimular diferentes áreas del TE del mono, observan una divergencia vertical de los ojos, junto a un movimiento conjugado y torsional de ambos globos oculares en la dirección del ojo hipotrópico. En 1979 Halmagyi publica el caso de una paciente con la triple reacción de inclinación óculo-cefálica tras estapedectomía, proponiendo que este síndrome es un mecanismo de compensación que aparece como consecuencia de una lesión en la vía utrículo-ocular. Hasta la actualidad, se han descrito y seguido con neuroimagen numerosos casos de reacción de inclinación ocular humana que han proporcionado una confirmación anatómica del sitio de la lesión [8].

\section{FISIOPATOLOGÍA}

La desviación oblicua es un reflejo natural que forma parte de una antigua respuesta filogenética de compensación a la inclinación lateral de la cabeza. Se observa en los animales con ojos laterales y serviría para mantener la alineación con el horizonte [1]. En el ser humano es un reflejo vestigial de escasa efectividad, pues tan sólo corrige un $10 \%$ el desplazamiento angular de la cabeza, siendo en este caso fundamental para la compensación, el movimiento torsional de contrarrotación ocular [13].

Este reflejo compensatorio funciona de la siguiente manera; si, por ejemplo, inclinamos la cabeza hacia el hombro derecho, se produce de forma refleja un estrabismo vertical (desviación oblicua) de los ojos, que consiste en un descenso del ojo izquierdo (hipotropía contralateral) y un ascenso del ojo derecho (hipertropía ipsilateral), con cilotorsión de ambos globos oculares hacia la izquierda (incliclotorsión del ojo derecho y exciclotorsión del izquierdo). Este mecanismo adaptativo de compensación ocular, nos permite inclinar la cabeza, sin que 
por ello el mundo a nuestro alrededor se mueva [6].

La vía neural del reflejo otolítico-ocular (arco reflejo del vestíbulo ocular) se origina en la macula de los órganos otolíticos, del utrículo y del sáculo y a través de un trayecto disináptico, las neuronas aferentes primarias procedentes del laberinto posterior se proyectan en neuronas de segundo orden a nivel de los núcleos vestibulares, desde donde parten señales que, a través del fascículo longitudinal medial contralateral, alcanzan los núcleos oculomotor y troclear. Pero también intervienen vías polisinápticas que tienen un papel crítico en la mediación de este reflejo y que conectan con el núcleo vestibular contralateral y con una extensa red de proyecciones dentro del cerebelo $[6,7]$.

El reflejo otolítico espinal (vía máculo-cólica) sería responsable de la inclinación de la cabeza $[7,10]$.

La desviación oblicua/reacción de inclinación (porque pueden aparecer juntas o manifestarse sólo la desviación oblicua), se observa clínicamente ante cualquier patología que interrumpa la vía máculo-ocular $[1,8,10,14]$. Hay que tener en cuenta que las lesiones a nivel de utrículo, nervio utricular o núcleo vestibular, van a provocar una reacción de inclinación óculo-cefálica ipsilateral al lado dañado, mientras que, por encima del núcleo vestibular, donde las fibras de esta vía refleja se decusan la desviación oblicua será del lado contrario. De tal manera que, un daño bulbo-protuberancial generaría una hipotropía del ojo del mismo lado y si se localiza en regiones más rostrales, a nivel mesencéfalo-diencefálico o núcleo intersticial de Cajal, la hipotropía sería del ojo contralateral $[4,6,7]$.

La desviación oblicua se suele asociar con un infarto del tronco cerebral, pero también ocurre en pacientes con patología cerebelosa o de la fosa posterior (esclerosis múltiple, tumores, trauma, abscesos, hemorragias, siringobulbia o procedimientos neuroquirúrgicos). Con mucha menos frecuencia es consecuencia de una lesión vestibular aguda unilateral (como en la neurectomía vestibular, laberintectomía, neuritis vestibular, herpes-zóster ótico y en algunos casos de estapedectomía, dehiscencia del CSS e inyección intratimpánica de gentamicina) $[4,8,12,14]$. También se ha descrito la desviación oblicua asociada a otras patologías, donde resulta difícil encontrar una explicación fisiopatológica, como en la epilepsia paroxística (en la que se supone una excitación desde los hemisferios cerebrales a porciones del núcleo vestibular, responsables del reflejo otolítico-ocular), Arnold-Chiari, cateterismo cardiaco, enfermedad de Creutzfelt-Jakob, encefalopatías multifocales, etc. [8].

\section{MANIFESTACIONES CLÍNICAS}

Los pacientes con desviación oblicua se quejan de una diplopía vertical (una imagen encima de otra) y a veces torsional (una imagen inclinada respecto a la otra). La ciclorotación se asocia ocasionalmente con una ilusión de inclinación del mundo visual [1].

Brandt y Dieterich (1991) explican 3 patrones de desviación oblicua, en función de la localización de la lesión:

- Cuando el daño se localiza en la mácula vestibular o nervio utricular, aparece una elevación de ambos ojos, pero está más alto el del lado sano. Se puede añadir un nistagmo que bate hacia el oído sano y una inclinación de la cabeza, de unos 10 a $30^{\circ}$, hacia el lado de la lesión. En estos casos, la desviación oblicua se suele recuperar en unas semanas.

- En las lesiones de la porción dorsolateral del bulbo (como en el síndrome de Wallenberg), con afectación del núcleo vestibular, se produce una elevación del ojo contrario, permaneciendo el del lado lesionado en su posición primaria.

- Finalmente, la patología mesencefálica pueden provocar simultáneamente la elevación de un ojo y el descenso del otro [15].

Tras su decusación, la vía otolítico-ocular asciende por el fascículo longitudinal medial, de forma que en la oftalmoplejia internuclear, se suele apreciar una desviación oblicua con hipertropía ipsilateral (el ojo más alto es el del lado afecto) [1-4].

En determinadas lesiones de la fosa posterior y cerebelosas la desviación oblicua es propiamente "oblicua" al mostrar alternancia del estrabismo vertical ocular en función de la posición de la mirada lateral. Usualmente el ojo que abduce es el hipertrópico [6]. En lesiones cercanas al núcleo intersticial de Cajal, algunos pacientes presentan una desviación oblicua que se manifiesta de forma paroxística, pudiéndose asociar a inclinación de la cabeza y donde se supone un mecanismo irritativo $[1,2,8,12]$.

Cuando la etiología reside en el sistema nervioso central, pueden añadirse otros hallazgos 
neurológicos como, nistagmo evocado por la mirada, oftalmoplejia internuclear, disartria, ataxia o hemiplejia [1-4].

\section{DIAGNÓSTICO}

La evaluación pretende reconocer una desviación oblicua y su posible asociación con la inclinación de la cabeza y la torsión ocular.

La desviación oblicua se explora pidiendo al paciente que mire de frente. El explorador nota la presencia de una falta de alineamiento vertical de ambos ojos en relación con la mirada horizontal. Una manera sencilla de evaluarlo es observando la simetría del reflejo corneal de la luz en ambos ojos (test de Hirschberg). Se debe diferenciar una asimetría facial de una desviación oblicua [16].

Sin embargo, la desviación oblicua se detecta mejor utilizando las técnicas oftalmológicas; de manera objetiva con el "cover test" o subjetivamente con el test de la varilla de Maddox [1].

COVER TEST: permite el diagnóstico de un estrabismo latente o manifiesto. Es necesaria la fijación.

- El cover test de un ojo: La presencia de una heterotropia (por ejemplo, estrabismo manifiesto) se observa en el ojo descubierto, cuando se cubre el otro. La heterotropia se define como una falta de alineación del eje visual, incluso con la fijación binocular. Al paciente se le pide fijar una diana. El explorador cubre un ojo y observa el movimiento corrector del ojo descubierto $[17,18]$. Si el ojo descubierto se mueve:

- Hacia fuera: esotropia.

- Hacia dentro: exotropia.

- Hacia abajo: hipertropía.

- Hacia arriba: hipotropía.

A continuación, se realiza el mismo examen en el otro ojo.

- $\quad$ El test cover/uncover de un ojo: se utiliza para probar la presencia de heteroforia (por ejemplo, un estrabismo latente). Se trata de una falta de alineamiento de los ejes oculares cuando se fija una diana con un solo ojo. Es importante realizar esta maniobra después de la anterior, para excluir primero una heterotropia. Se cubre un ojo durante unos $10 \mathrm{~s}$, y entonces se descubre. Se observa el posible movimiento corrector que aparece en el ojo previamente cubierto. Si se mueve:

- Hacia fuera: esoforia.

- Hacia dentro. exoforia.
- Hacia abajo: hiperforia

- Hacia arriba: hipoforia

- El test de cover altenante: sirve para evaluar el desalineamiento máximo de los ejes oculares, tanto en una tropia como en una foria. Es especialmente útil en la evaluación de la desviación oblicua, donde miraremos el componente correctivo vertical cuando el recubrimiento ocular pasa de un ojo a otro. A veces resulta difícil de distinguir si se asocia a nistagmo [17].

- Mediante el cristal rojo de la varilla de Maddox, al disociarse las imágenes de ambos ojos, el paciente verá una imagen sobre otra, indicándonos la desalineación vertical [1].

También analizaremos el efecto que sobre la desviación oblicua tiene la posición del ojo en la órbita, para excluir parálisis del IV par. Típicamente la parálisis del IV par es una desalineación vertical que se incrementa cuando el ojo afectado es llevado hacia abajo y a la línea media, mientras que en la desviación oblicua el estrabismo vertical cambia muy poco en las distintas posiciones de la mirada. Pero en ocasiones esta diferenciación es compleja, precisándose de un diagnóstico oftalmológico especializado $[1,2,4]$.

La torsión ocular es difícil de detectar clínicamente, pero si tiene una suficiente magnitud, puede ser apreciada como una inclinación de una línea imaginaria horizontal que conecta la mácula óptica con la papila del nervio óptico. Al contrario del movimiento fisiológico de inclinación de la cabeza, en donde los ojos giran hacia el oído más elevado, la torsión que aparece consecuencia de una lesión patológica se produce hacia el más bajo (hacia donde se inclina la cabeza) [1].

La inclinación de la cabeza puede aparecer tanto en lesiones periféricas como centrales, donde es posible que sea la consecuencia de un predominio de la vía utrículo-mesencefálica contralateral o de una inhibición de la ipsilateral $[6,12]$.

\section{CONCLUSIONES}

En presencia de una desviación oblicua, buscaremos otros síntomas/signos neurológicos, que junto a las pruebas de imagen nos permitan descartar patología neurológica central. Tendremos en cuenta que podría tratarse del 
signo fundamental para descartar un infarto tronco-encefálico en un paciente con vértigo agudo $[6,8]$. En el contexto de una vestibulopatía periférica la evolución de la triple reacción de inclinación óculo-cefálica, es un índice de compensación vestibular [1,3-5,7].

\section{BIBLIOGRAFÍA}

1. Carmona, Sergio; Asprella Libonati G. Una aproximación fisiopatológica a la evaluación clínica del paciente con vértigo. En: Neuro-otologia. 3a ed. Buenos Aires: Libreria Akadia editorial; 2013. p. 19-25.

2. Wong AMF. Understanding skew deviation and a new clinical test to differentiate it from trochlear nerve palsy. J AAPOS. 2010;14(1):61-7.

3. Schlenker M, Mirabella G, Goltz HC, Kessler P, Blakeman AW, Wong AMF. The Linear Vestibulo-Ocular Reflex in Patients with Skew Deviation. Investig Opthalmology Vis Sci. 2009;50(1):16874.

4. Bateman JA, Chang A, Capo-Aponte J. Cerebellar ischemia manifesting as vertical diplopia: a case study on skew deviation. Mil Med. 2015;180(1):16873.

5. Zee DS. Considerations on the mechanisms of alternating skew deviation in patients with cerebellar lesions. J Vestib Res. 1996;6(6):395401.

6. Moguel-Ancheita S, Castellanos-Pérez Balde CG, Orozco-Gómez LP. Desviación oblicua. Diagnóstico estrabológico y alternativas de tratamiento. Cir Cir. 2009;77(4):267-73.

7. Wong AMF. New understanding on the contribution of the central otolithic system to eye movement and skew deviation. Eye. 2015;29(2):153-6.

8. Brodsky MC, Donahue SP, Vaphiades M, Brandt T. Skew deviation revisited. Surv Ophthalmol. 2006;51(2):105-28.
9. Brandt T, Dieterich M. Pathological eyehead coordination in roll: tonic ocular tilt reaction in mesencephalic and medullary lesions. Brain. 1987;110 (Pt 3):649-66.

10. Wertheimer NP, Pinel B. Otolith dysfunction in skew deviation after brain stem lesions. J Vestib Res. 2000;10:179-92.

11. Brandt $\mathrm{T}$, Dieterich $\mathrm{M}$. Vestibular syndromes in the roll plane: topographic diagnosis from brainstem to cortex. Ann neurol. 1994;36(3):33747.

12. Hernowo A, Eggenberger E. Skew deviation: clinical updates for ophthalmologists. Curr Opin Ophthalmol. 2014;25(6):485-7.

13. Baloh, RW; Kerber K. Clinical neurophysiology of the vestibular system. 4. ${ }^{a}$ ed. New York: Oxford University Press; 2011. 455 p.

14. $\mathrm{Ng} \mathrm{D,} \mathrm{Fouladvand} \mathrm{M,} \mathrm{Lalwani} \mathrm{AK.} \mathrm{Skew}$ deviation after intratympanic gentamicin therapy. Laryngoscope. 2011;121(3):492-4.

15. Brandt TH, Dieterich M. Different types of skew deviation. J Neurol Neurosurg Psychiatry. junio de 1991;54(6):549-50.

16. McCaslin, DL; Dundas, JA; Jacobson G. Balance function assessment and management. En: The bedside assessment of the vestibular system. 1. ${ }^{\mathrm{a}}$ ed. United Kingdom: Plural publishing; 2008. p. 63-97.

17. Brandt, $\mathrm{T}$; Dieterich, $\mathrm{M}$; Strupp $\mathrm{M}$. Vertigo and Dizziness. 2. ${ }^{a}$ ed. London: Springer-Verlag; 2013. 189 p.

18. Kennedy SA, Noble J, Wong AMF. Esotropia. Can Med Assoc J. 2012;184(11):1279-1279. 